\title{
Combinatorial aspects of Löwenstein's rule
}

\author{
J.-G. Eon ${ }^{1}$, M. Moreira de Oliveira Jr² \\ ${ }^{1}$ Instituto de Química, Universidade Federal do Rio de Janeiro, Avenida Athos da Silveira Ramos, 149 Bloco A, Cidade Universitária, \\ Rio de Janeiro 21941-909, Brazil, ${ }^{2}$ Instituto de Ciências Exatas, Matemática Pura, Universidade Federal Rural do Rio de Janeiro, \\ P1-90-4, Seropédica, Rio de Janeiro 23890-000, Brazil
}

jgeon@iq.ufrj.br

According to Löwenstein's rule [1], Al-O-Al bridges are forbidden in the aluminosilicate framework of zeolites. A graph-theoretical interpretation of the rule, based on the concept of independent sets, was proposed by Klee [2] and reviewed by Eon [3]. It was shown that one can apply the vector method to the associated periodic net and define a maximal $\mathrm{Al} /(\mathrm{Al}+\mathrm{Si})$ ratio for any aluminosilicate framework following the rule; this ratio was called the independence quotient of the net. This presentation deals with practical issues regarding the calculation of the independence quotient of mainly 2-periodic nets and the possible existence of disordered structures with this ratio.

We first show that applying Proposition Calculus to the determination of independent sets in finite graphs leads to introducing a multivariate polynomial, called the independence polynomial. This polynomial can be calculated in an automatic way and provides the list of all maximally independent sets of the graph, hence also the value of its independence quotient. Some properties of this polynomial are discussed; the independence polynomials of some simple graphs, such as short paths or cycles, are determined as examples of calculation techniques.

The determination of the independence quotient of a periodic net requires finding a subgroup of the translation group of the net for which the quotient graph and a fundamental transversal have the same independence quotient. See Fig. 1 for an illustration based on the hbt net, with independence quotient of 4/7; the only maximally independent set in the quotient graph and in the transversal associated to a primitive unit cell is shown in red. In most nets, however, a non-trivial translation subgroup has to be found. We show that this subgroup should be chosen to eliminate every cycle in the quotient graph that is shorter than structural cycles, or rings, of the net. Several examples are then analysed, which show that the choice of the fundamental transversal is critical; no rule, however, can yet be formulated concerning this choice.
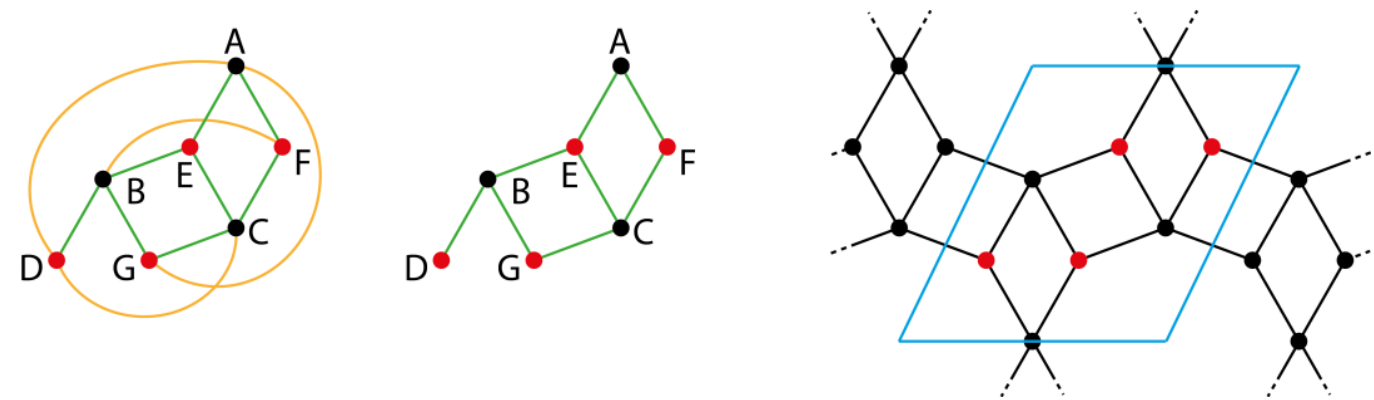

Figure 1. The quotient graph (left) and a fundamental transversal (middle) of hbt (right).

The existence of disordered materials with substitution ratio equal to the independence quotient of the respective periodic net is related to the multiplicity of solutions for maximally independent sets of its quotient graph. Some examples are analysed, summarizing different possible situations in 2-periodic nets. The disorder can be complete in two directions or partial and limited to one direction.

[1] Löwenstein, W. (1954). Am. Mineral. 39, 92.

[2] Klee, W. E. (1974). Z. Kristallogr. 140, 154.

[3] Eon, J.-G. (2016). Struct. Chem. 27, 1613.

Keywords: Löwenstein's rule; periodic nets; quotient graphs; independent sets

J.-G. Eon thanks CNPq, Conselho Nacional de Desenvolvimento e Pesquisa of Brazil, for support during the preparation of this work. 